\title{
The integral trees with spectral radius 3
}

\author{
A. E. Brouwer \& W. H. Haemers
}

2007-09-11

\begin{abstract}
There are 11 integral trees with largest eigenvalue 3 .

\section{$1 \quad$ Integral trees}

A finite graph is called integral if the spectrum of its adjacency matrix has only integral eigenvalues. A tree is a connected undirected graph without

In [1] a table is given of the integral trees on at most 48 vertices. (There are only 25 of these, showing that integral trees are rare objects.)

The nicest result about integral trees is that by Watanabe [13] that says that an integral tree different from $K_{2}$ does not have a complete matching.

All 'starlike' integral trees, that is, all integral trees with at most one vertex of degree larger than 2, were given by Watanabe and Schwenk [14].

All integral trees of diameter at most 3 were given in $[14,5]$. See also

Several people have worked on constructing integral trees with large diameter, and examples with diameters $0-8$ and 10 are known, see $[14$, $10,3,9,7,8]$. It is unknown whether integral trees can have arbitrarily large diameter.
\end{abstract} cycles. $[11,3]$.

\section{$1.1 \quad$ Names}

Here we are interested in classification, and in order to indicate which tree is meant, some notation is needed. Given a tree, pick some vertex and call it the root. Now walk along the tree (depth-first), starting at the root, and when a vertex is encountered for the first time, write down its distance to the root. The sequence of integers obtained is called a level sequence for the tree. A tree is uniquely determined by any level sequence. The parent of a vertex labeled $m$ is the last vertex encountered earlier that was labeled $m-1$.

For example, the graph $K_{1,4}$ gets level sequence 01111 if the vertex of degree 4 is chosen as root, and 01222 otherwise.

We use exponents to indicate repetition: 01111 can be written $01^{4}$ and 0121212 as $0(12)^{3}$.

\subsection{Classification}

One can try to classify integral trees by their largest eigenvalue $\lambda_{\max }$.

The only example with $\lambda_{\max }=0$ is $K_{1}$, with spectrum 0 .

The only example with $\lambda_{\max }=1$ is $K_{2}$, with spectrum $1,-1$. 
There are three integral trees with $\lambda_{\max }=2$, namely 01111 (on 5 vertices, with spectrum $2,0^{3},-2$ ) and 012211 (on 6 vertices, with spectrum $2,1,0^{2},-1,-2$ ) and 0121212 (on 7 vertices, with spectrum $2,1^{2}, 0$, $\left.(-1)^{2},-2\right)$. Here exponents denote eigenvalue multiplicities.

(Since all graphs with largest eigenvalue at most 2 are known, the above follows by simple inspection. See also $§ 2.7$ below.)

In this note we find all integral trees with $\lambda_{\max }=3$. The table below gives a serial number, the number of vertices $n$, the diameter $d$, a level sequence and the nonnegative part of the spectrum. (Note that trees are bipartite, and hence have a spectrum that is symmetric w.r.t. 0.)

\begin{tabular}{lllll}
$\#$ & $n$ & $d$ & graph & spectrum \\
\hline 1 & 10 & 2 & $01^{9}$ & $3,0^{8}$ \\
2 & 14 & 3 & $012^{6} 1^{6}$ & $3,2,0^{10}$ \\
3 & 17 & 4 & $0(12)^{8}$ & $3,1^{7}, 0$ \\
4 & 17 & 4 & $012^{7}(12)^{4}$ & $3,2,1^{3}, 0^{7}$ \\
5 & 19 & 4 & $012^{5}(12)^{6}$ & $3,2,1^{5}, 0^{5}$ \\
6 & 25 & 5 & $01\left(23^{4}\right)^{3} 22(12)^{3}$ & $3,2^{3}, 1^{3}, 0^{11}$ \\
7 & 26 & 4 & $0\left(12^{4}\right)^{5}$ & $3,2^{4}, 0^{16}$ \\
8 & 31 & 6 & $0123^{3}\left(1(23)^{3}\right)^{2}\left(12^{4}\right)^{2} 1$ & $3,2^{4}, 1^{5}, 0^{11}$ \\
9 & 31 & 6 & $0\left(123^{3}\right)^{3}\left(12^{4}\right)^{3}$ & $3,2^{5}, 1,0^{17}$ \\
10 & 37 & 6 & $0\left(1(23)^{3}\right)^{5} 1$ & $3,2^{4}, 1^{11}, 0^{5}$ \\
11 & 37 & 6 & $0\left(123^{3}\right)^{2}\left(1(23)^{3}\right)^{3} 12^{4}$ & $3,2^{5}, 1^{7}, 0^{11}$
\end{tabular}

Theorem Let $T$ be a tree with integral eigenvalues and largest eigenvalue 3. Then $T$ is one of the above 11 graphs.

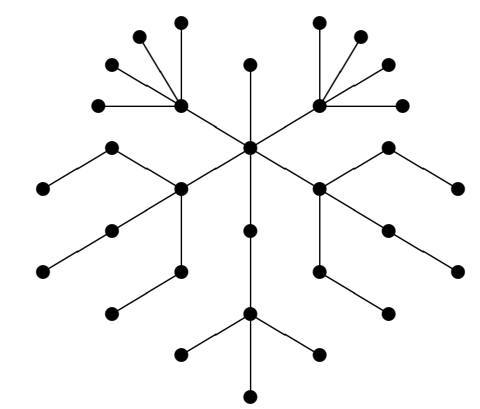

\#8: $0123^{3}\left(1(23)^{3}\right)^{2}\left(12^{4}\right)^{2} 1$.

\subsection{A family of examples}

Of the above 11 examples, 8 have a spectral center (cf. $\S 2.6$ below) that is a single vertex. Let us give a uniform description of these.

Consider the tree $01^{a}(12)^{b}(12222)^{c}(12333)^{d}(1232323)^{e}$. It has $n=$ $1+a+2 b+5 c+5 d+7 e$ vertices, and spectrum $0^{a+3 c+3 d+e-1}( \pm 1)^{b+2 e-1}$ $( \pm 2)^{c+d+e-1}$ plus 6 more eigenvalues, roots of $x^{2}\left(x^{2}-1\right)\left(x^{2}-4\right)=a\left(x^{2}-\right.$ 1) $\left(x^{2}-4\right)+b x^{2}\left(x^{2}-4\right)+c x^{2}\left(x^{2}-1\right)+d\left(x^{2}-1\right)\left(x^{2}-3\right)+e\left(x^{2}-1\right)^{2}$.

(This follows by direct computation of the characteristic polynomial. These last 6 eigenvalues belong to the eigenvectors that are nonzero at the top vertex.)

The tree is integral in the cases $01^{a}$ with $a=t^{2}$, and $0(12)^{b}$ with $b=t^{2}-1$, and $0(12222)^{c}$ with $c=t^{2}-4$, and $b=0, c=3 a+2 d-3$, $e=t^{2}-4 a-3 d$, where $t$ is the largest eigenvalue.

For $t=3$ we find examples \#1, 3,6-11. Many of the integral trees in this family, and in particular \#8 and \#11, seem to be new. 


\section{Generalities}

We start describing some tools we need. Everything is very well known, except possibly for the concept of 'spectral center' of a tree.

\subsection{Perron-Frobenius}

Let $T$ be a connected graph with largest eigenvalue $\lambda$. Then $\lambda$ has multiplicity 1 , and any proper subgraph of $T$ (induced or not) with at least one vertex has largest eigenvalue strictly less than $\lambda$.

\subsection{Interlacing}

Let $T$ be a graph with eigenvalues $\theta_{1} \geq \theta_{2} \geq \ldots \geq \theta_{n}$, and let $x$ be a vertex of $T$. Let $T \backslash x$ have eigenvalues $\eta_{1} \geq \ldots \geq \eta_{n-1}$. Then $\theta_{1} \geq \eta_{1} \geq$ $\theta_{2} \geq \eta_{2} \geq \ldots \geq \eta_{n-1} \geq \theta_{n}$.

\subsection{Stars}

The star $K_{1, m}$ has spectrum $\sqrt{m}, 0^{m-1},-\sqrt{m}$. In particular, if a graph has a vertex of valency $m$, then its largest eigenvalue is at least $\sqrt{m}$.

\subsection{Godsil's Lemma}

Let $T$ be a tree and $\theta$ an eigenvalue of multiplicity $m>1$. Let $P$ be a path in $T$. Then $\theta$ is eigenvalue of $T \backslash P$ with multiplicity at least $m-1$. $([4])$

\subsection{Eigenvalue components}

Given an eigenvalue $\lambda$ of a tree $T$, let $Z=Z(\lambda)$ be the set of vertices where all $\lambda$-eigenvectors vanish. An eigenvalue component of $T$ for the eigenvalue $\lambda$ is a connected component of $T \backslash Z$. Each eigenvalue component has $\lambda$ as an eigenvalue of multiplicity 1 . Let $Z_{0}=Z_{0}(\lambda)$ be the subset of $Z$ consisting of the $z \in Z$ with a neighbour in $T \backslash Z$. If $T \backslash Z$ has $p$ connected components, and $q=\left|Z_{0}\right|$, then the eigenvalue $\lambda$ of $T$ has multiplicity $p-q$. If $\lambda$ is the $j$-th largest eigenvalue of $T$, then $0 \leq\left|Z_{0}\right| \leq j-1$.

\subsection{The spectral center of a tree}

There are various combinatorial concepts 'center' for trees. One has the center/bicenter and the centroid/bicentroid. Here we define a concept of center using spectral methods.

Proposition 1 Let $T$ be a tree (with at least two vertices) with second largest eigenvalue $\lambda$. There is a unique minimal subtree $Y$ of $T$ such that no connected component of $T \backslash Y$ has largest eigenvalue larger than $\lambda$. We have $1 \leq|Y| \leq 2$ and $Z_{0}(\lambda) \subseteq Y$.

We call the set $Y$ the spectral center of $T$.

Proof If for some vertex $y$ all connected components of $T \backslash y$ have largest eigenvalue at most $\lambda$, then pick $Y=\{y\}$. Otherwise, for each vertex $y$ of $T$ there is a unique neighbour $y^{\prime}$ in the unique component of $T \backslash$ $y$ that has largest eigenvalue more than $\lambda$. Since $T$ is finite, we must have $y^{\prime \prime}=y$ for some vertex $y$. Now pick $Y=\left\{y, y^{\prime}\right\}$. Clearly $Y$ has 
the stated property and is minimal. Uniqueness is clear in case $|Y|=$ 2. If no connected component of $T \backslash Y_{1}$ has largest eigenvalue larger than $\lambda$, and $Y=\{y\}$, then consider the eigenvalue components of $\lambda$. If $Z$ is nonempty (in particular, if $\lambda$ has multiplicity more than 1 ), then necessarily $Z_{0}=\{y\}$, and $Y_{1}$ must contain $y$, and by minimality $Y_{1}=Y$. If $\lambda$ has multiplicity 1 and $Z$ is empty, then there is a unique edge $e=p q$ such that the unique $\lambda$-eigenvector has different signs on $p$ and $q$, and both components of $T \backslash e$ have largest eigenvalue strictly larger than $\lambda$, so that $Y$ must contain both endpoints of $e$.

One can be slightly more precise. In fact $Y=Z_{0}$ when $Z$ is nonempty, and in this case $|Y|=1$. Otherwise $|Y|=2$ and $Y$ contains the two endpoints of the edge on which the unique $\lambda$-eigenvector changes sign.

\subsection{The trees with largest eigenvalue at most 2}

The trees (integral or not) with largest eigenvalue less than 2 are the Dynkin diagrams $A_{n}, D_{n}, E_{6}, E_{7}, E_{8}$. The trees (integral or not) with largest eigenvalue 2 are the extended Dynkin diagrams $\tilde{D}_{m}, \tilde{E}_{6}, \tilde{E}_{7}, \tilde{E}_{8}$. Every tree with largest eigenvalue larger than 2 contains a subtree with largest eigenvalue 2. (This is due to Smith, see, e.g., [2], Theorem 3.2.5.)

These traditional Dynkin diagram names mean the following: $A_{n}$ is the path with $n$ vertices, that is, has level sequence $0,1, \ldots, n-1(n \geq 1)$. $D_{n}$ has level sequence $0,1,1,1,2, \ldots, n-3(n \geq 4)$, so that $D_{4}$ is 0111 and $D_{5}$ is $01112 . E_{6}$ is $011212, E_{7}$ is $0112123, E_{8}$ is $01121234 . \tilde{D}_{4}$ is 01111 (that is, is $K_{1,4}$ ), $\tilde{D}_{m}$ has level sequence $0,1,1,1,2, \ldots, n-4, n-3, n-3$ $(n \geq 5)$, so that $\tilde{D}_{5}$ is $011122 . \tilde{E}_{6}$ is $0121212, \tilde{E}_{7}$ is $01123123, \tilde{E}_{8}$ is 011212345 .

There are 5 integral trees with largest eigenvalue at most 2, namely $A_{1}, A_{2}, \tilde{D}_{4}, \tilde{D}_{5}, \tilde{E}_{6}$. They have $1,2,5,6,7$ vertices, and diameter 0,1 , $2,3,4$, respectively.

Every nonintegral tree with largest eigenvalue at most 2 has a nonintegral eigenvalue between 1 and 2 . There are 11 trees with largest eigenvalue at most 2 and a unique nonintegral eigenvalue between 1 and 2, namely $A_{3}, A_{4}, A_{5}, D_{4}, D_{5}, E_{6}, \tilde{D}_{6}, \tilde{D}_{7}, \tilde{D}_{8}, \tilde{E}_{7}, \tilde{E}_{8}$, They have $3,4,5,4,5,6,7$, $8,9,8,9$ vertices, and diameter $2,3,4,2,3,4,4,5,6,6,7$, respectively.

\section{Proof of the theorem}

It is straightforward to check that the 11 graphs have the spectrum given in the table. Conversely, a computer search shows that there are no other examples. Below we show how to reduce the search space so that the computer search becomes feasible.

\subsection{Finitely many examples}

Consider a tree $T$ with no other eigenvalues than $\pm 3, \pm 2, \pm 1,0$.

The diameter of a connected graph is smaller than the number of distinct eigenvalues. It follows that $T$ has diameter at most 6 .

The 9 -star $K_{1,9}$ has largest eigenvalue 3 (it is \#1 on the list), and a graph that strictly contains it has larger eigenvalue. Thus, if $T$ is not $K_{1,9}$ then its maximum valency is at most 8 . 
Now that both diameter and valency have been bounded, there are only finitely many possibilities.

\subsection{Vertices of high degree}

By interlacing, if $x$ is any vertex of $T$, then at most one component of $T \backslash x$ has largest eigenvalue larger than 2 . In particular, at most one component of $T \backslash x$ has largest degree more than 4 . It follows that two vertices of degree more than 5 must be adjacent, and there can be at most two such vertices. The largest eigenvalue of the bi-star $012^{r} 1^{m}$ obtained by adding an edge joining the central vertices of $K_{1, m}$ and $K_{1, r}$ is the largest root of the polynomial $\left(X^{2}-m\right)\left(X^{2}-r\right)-X^{2}$. It follows that if this bi-star is an induced subgraph (i.e., if there are two adjacent vertices of degrees $m+1$ and $r+1)$ then $(9-m)(9-r)-9 \geq 0$ with equality only when this bi-star is all of $T$. Consequently, a neighbour of a vertex of degree 8 has degree at most 5 , and a neighbour of a vertex of degree 7 has degree at most 7 , and two adjacent vertices of degree 7 occur only in $012^{6} 1^{6}$, graph \#2 on the list.

\subsection{Components of a tree minus a point}

Let $T$ be an integral tree with largest eigenvalue 3 , and $x$ a vertex of $T$. Since the spectrum of $T \backslash x$ is the union of the spectra of its connected components, it follows by interlacing that among the components of $T \backslash x$ there is at most one that has a (unique) nonintegral eigenvalue between 1 and 2 , and at most one that has an eigenvalue larger than 2 .

In particular, all components of $T \backslash x$ are one of $A_{1}, A_{2}, \tilde{D}_{4}, \tilde{D}_{5}, \tilde{E}_{6}$, except for at most one with largest eigenvalue larger than 2 , and at most one that is nonintegral but has largest eigenvalue at most 2 and a unique nonintegral eigenvalue between 1 and 2 .

None of $\tilde{D}_{7}, \tilde{D}_{8}, \tilde{E}_{7}$ can occur as component of $T \backslash x$ when there are other components not reduced to a single point. Indeed, the point of attachment $w$ in such a component must be the middle vertex (or one of the two middle vertices), otherwise $T$ gets diameter larger than 6 . But then $T \backslash w$ has two nonintegral eigenvalues between 1 and 2, impossible. This argument applied to $\tilde{D}_{6}$ shows that it cannot be attached at the middle node, otherwise $T$ gets eigenvalue $\sqrt{2}$.

Let $Y$ be the spectral center of $T$. If $Y=\{y\}$, then $n \leq 1+56=57$ since $y$ has valency at most 8 and all components of $T \backslash y$ have at most 7 vertices. Otherwise $Y=\left\{y, y^{\prime}\right\}$, and $n \leq 2+77=79$ since we saw that the sum of the valencies of two neighbours is at most 13 .

\subsection{Diameter}

Let $S_{1}, S_{2}$ be disjoint subtrees of $T$ at mutual distance $e$ and with respective diameters $d_{1}, d_{2}$. Then $T$ has diameter at least $\left\lceil d_{1} / 2\right\rceil+e+\left\lceil d_{2} / 2\right\rceil$, so this expression is at most 6 . That means that the last estimate can be improved: if all components of $T \backslash\left\{y, y^{\prime}\right\}$ have largest eigenvalue at most 2 , then $n \leq 2+49+20=71$.

\subsection{All possible spectra}

Consider a tree $T$ on $n$ points, with adjacency matrix $A$ with spectrum $\pm 3,( \pm 2)^{a},( \pm 1)^{b}, 0^{c}$, where exponents denote multiplicities. Since the 
number of eigenvalues equals $n$, we have $n=2 a+2 b+c+2$.

The number of closed walks of length $s$ in $T$ equals $\operatorname{tr} A^{s}$. In particular we find $\operatorname{tr} A^{2}=2(n-1)$ (twice the number of edges), and it follows that $9+4 a+b=n-1$, that is, $8+2 a=b+c$.

Thus, the spectrum is given by the pair $(n, a)$. Now that $n$ is bounded, we can enumerate all pairs $(n, a)$ with $n \leq 71$ and $b=n-4 a-10 \geq 0$ and $c=6 a+18-n \geq 0$. There are 251 of those.

\subsection{Eigenvalue components}

Let 2 be an eigenvalue of the tree $T$ with multiplicity $a$. Let $Z$ be the set of vertices where all 2-eigenvectors vanish, and let $Z_{0}$ be the subset of the $z \in Z$ with a neighbour in $T \backslash Z$. Then $T \backslash Z$ has $p=a+q$ connected components, where $q=\left|Z_{0}\right|$. Each connected component has size at least 5 , so $n-q \geq n-|Z| \geq 5 p$. It follows that if $a>1$, then $q \geq 1$ and $n-1 \geq 5(a+1)$. Now 198 spectra are left.

If $C, D$ are two components of $T \backslash Z$ and $x$ a vertex nonadjacent to both, then $x$ cannot separate $C$ and $D$, since that would give two components of $T \backslash x$ both with largest eigenvalue larger than 2 . It follows that $q \leq 2$, and if $q=2$ then the two vertices in $Z_{0}$ are neighbours.

If there is one component of $T \backslash Z$ with largest eigenvalue greater than 2 then $q=1$. Every other component is one of $\tilde{D}_{m}(4 \leq m \leq 6), \tilde{E}_{6}$.

\begin{tabular}{llllllll} 
comp & diam & size & ev in $(2,3)$ & ev in $(1,2)$ & val 4 & val 3 & val 2 \\
\hline$\tilde{D}_{4}$ & 2 & 5 & 0 & 0 & 1 & 0 & 0 \\
$\tilde{D}_{5}$ & 3 & 6 & 0 & 0 & 0 & 2 & 0 \\
$\tilde{D}_{6}$ & $4+$ & 7 & 0 & 1 & 0 & 2 & 1 \\
$\tilde{E}_{6}$ & 4 & 7 & 0 & 0 & 0 & 1 & 3 \\
$?$ & $?$ & $\geq 11$ & 1 & $?$ & $?$ & $?$ & $?$
\end{tabular}

\subsection{Matchings}

By Watanabe [13], the tree $T$ does not have a complete matching. But the characteristic polynomial of $T$ equals $\phi_{T}(X)=\sum_{k}(-1)^{k} a_{2 k} X^{n-2 k}$, where $a_{2 k}$ is the number of ways one can choose $k$ pairwise disjoint edges in $T$. That means that $\phi_{T}(X)$ has zero constant term, so that there is an eigenvalue 0 , and $c \neq 0$. Now 189 spectra are left.

\subsection{Degree sequences}

Let $d_{x}$ denote the degree of the vertex $x$. Then $\sum d_{x}=2(n-1)$, twice the number of edges. And $\operatorname{tr} A^{4}=2 \sum d_{x}^{2}-2(n-1)$ so that $\sum d_{x}^{2}=$ $81+16 a+b+n-1=90+20 a+2 b=70+12 a+2 n$. We can solve this for all possible degree sequences $\left(d_{x}\right)$, recalling that there are at most two vertices of degree more than 5 . If there is no possible sequence, the triple can be dropped. Now 185 spectra are left.

If the largest degree is smaller than $a+1$ then $q=2$ and $n-2 \geq 5(a+2)$. In this case the sum of the two largest degrees must be at least $a+4$. Now 141 spectra are left. 


\subsection{Eigenvalue components (2)}

Each component of size 5 of $T \backslash Z$ contains a point of degree 4 or 5 . Components for which no such point is available have size at least 6 . Now 109 spectra are left.

The number of components is $p=a+q$, and all these components contain a point of valency 3 or 4 , after attachment 3 or 4 or 5 . So, there must at least be $p$ points of valency 3,4 , or 5 , and at least $p+q$ points of valency at least 3 . Now 92 spectra are left.

The point of attachment of a component $\tilde{E}_{6}$ cannot be one of its leaves, since that would give $T$ too large a diameter. It follows that if a component does not contain points of valency 4 or 5 , then it contains at least two points of valency 3 . Now 76 spectra are left.

(Note that here an unknown component of largest eigenvalue larger than 2 does not harm: if it has no vertices of valency 4 or more, and at most a single vertex of valency 3 , then the component is topologically a star, and if it has eigenvalue 2 that must be the largest eigenvalue because the eigenvector has constant sign. And if there is a vertex of valency 6 or more, then it must be the point of attachment (otherwise $T$ gets two eigenvalues larger than 2), and therefore unique, and now again the 2eigenvector has constant sign, contradiction.)

If a component does not contain points of valency 3 or at least 5 , then it must also contain a point of valency 2. (A $\tilde{D}_{4}$ component has a point of attachment: either its center, which then gets valency 5 , or a leaf, which then gets valency 2.) Now 73 spectra are left.

\subsection{Walks of length 6}

$\operatorname{tr} A^{6}$ counts the number of closed walks of length 6 . We find

$$
3^{6}+2^{6} a+b=\sum d_{x}^{3}-3 \sum d_{x}^{2}+2(n-1)+3 \sum_{e} d_{x} d_{y}
$$

where the last sum is over all unordered edges $e=x y$. Given the degree sequence, this allows us to compute $\sum_{e} d_{x} d_{y}$. The maximal possible value of $\sum_{e} d_{x} d_{y}$ is obtained by ordering the vertices by decreasing degree, and building the tree by starting with the vertex of highest degree, and each step attaching a new vertex of highest possible degree to the vertex of highest degree in the tree that still has room left. If this maximum is smaller than the computed $\sum_{e} d_{x} d_{y}$, we can discard the degree sequence. Now 59 spectra are left.

\subsection{Matchings (2)}

As already remarked, the characteristic polynomial of $T$ equals $\phi_{T}(X)=$ $\sum_{k}(-1)^{k} a_{2 k} X^{n-2 k}$, where $a_{2 k}$ is the number of ways one can choose $k$ pairwise disjoint edges in $T$. That means that the product of the nonzero eigenvalues is the number of ways of chosing $m$ disjoint edges, where $n-2 m=c$ is the multiplicity of the eigenvalue 0 .

Thus, the maximum number of pairwise disjoint edges equals $m=$ $a+b+1$. The number of non-leaves must be at least this much since no edge contains two leaves. This provides an upper bound $9+3 a$ for the number of vertices of degree 1 . In case of equality, the number of ways to choose $m$ disjoint edges is the product of the number of leaves attached to the non-leaf vertices. 
For example, if $n=16$ and the positive spectrum is $3,2,1^{2}, 0^{8}$, and the degree sequence is $12 \times 11 \times 21 \times 31 \times 51 \times 8$, then the product of the nonzero eigenvalues is 36 , and this is the product of 4 factors, namely $1,1 . .2,1 . .4,1 . .7$. The only possibility is $1 * 2 * 3 * 6$, so that the 'internal graph', after removal of the 12 leaves, is a path. But if the internal graph is a path, no nonzero eigenvalue can have multiplicity larger than 1 , since the eigenvector is uniquely determined. So, no such graph exists.

\subsection{Finish}

At this point 58 spectra are left, all with $n \leq 45$. A computer search for all trees on at most 45 vertices, with diameter at most 6 , maximum valency at most 8 , and integral spectrum now finishes the job.

\section{Application of Godsil's Lemma}

It is possible to do everything by hand, avoiding the use of a computer. (But the proof becomes long and tedious.)

The first tool to use in such a hand proof is Godsil's Lemma. It allows one to dispose of the cases of diameter 5 and 6 . Let us show here a small fragment.

Suppose $T$ has diameter 6 , the maximum possible value. Then $a, b, c$ are nonzero. Let $P$ be a path of length 6 , that is, with 7 vertices. By Godsil's Lemma, the forest $T \backslash P$ has spectrum $( \pm 2)^{a-1},( \pm 1)^{b-1}, 0^{c-1}$, and hence is integral, and each tree in that forest is one of $A_{1}, A_{2}, \tilde{D}_{4}, \tilde{D}_{5}$, $\tilde{E}_{6}$. The number of trees in that forest is the number of vertices minus the number of edges, that is, is $(n-7)-(4 a-4+b-1)=8$.

Suppose that at least one of the components of $T \backslash P$ is $\tilde{D}_{5}$, or $\tilde{E}_{6}$, or $\tilde{D}_{4}$ (attached at a leaf). Then such a component must be joined to the middle vertex $p$ of $P$, otherwise the diameter would become larger than 6. Now there are three paths $P_{i}(i=1,2,3)$ of length 3 starting at $p$ such that for $i \neq j$ the union $P_{i} \cup P_{j}$ is a path of length 6 .

Let $\phi(T)$ denote the characteristic polynomial of a tree $T$. Let $Q_{i}$ be the connected component of $T \backslash p$ containing $P_{i} \backslash p$. Since the spectrum of $T \backslash\left(P_{i} \cup P_{j}\right)$ is the same for all choices of $i \neq j$, it follows that $\phi\left(Q_{i}\right) / \phi\left(Q_{i} \backslash\right.$ $\left.P_{i}\right)$ is the same for all $i$.

Now $\phi\left(Q_{i}\right) / \phi\left(Q_{i} \backslash P_{i}\right)$ in the three cases $\tilde{D}_{5}, \tilde{E}_{6}, \tilde{D}_{4}$ (attached at a leaf) equals $\left(X^{2}-4\right)\left(X^{2}-1\right) / X,\left(X^{2}-4\right) X,\left(X^{2}-4\right) X$.

If one of the $Q_{i}$ is of shape $\tilde{D}_{5}$, then all are, and $T \backslash p$ has spectrum $( \pm 2)^{a+1},( \pm 1)^{b+1}, 0^{c-3}$, impossible, since the multiplicity of 0 cannot decrease by 3 when a single point is removed.

So, only the shapes $\tilde{D}_{4}$ and $\tilde{E}_{6}$ occur, and $T \backslash p$ has spectrum $( \pm 2)^{a+1}$, $( \pm 1)^{b-1}, 0^{c+1}$, and has $(n-1)-(4 a+4+b-1)=6$ components.

Let $T \backslash p$ have $n_{1}, n_{2}, n_{5 a}, n_{5 b}, n_{7}$ components of shape $A_{1}, A_{2}, \tilde{D}_{4}$ (attached at the center), $\tilde{D}_{4}$ (attached at a leaf), $\tilde{E}_{6}$, respectively. Then $n_{1}+n_{2}+n_{5 a}+n_{5 b}+n_{7}=6$. The condition for $T$ to have eigenvalue 3 is $5 n_{1}+\frac{45}{8} n_{2}+9 n_{5 a}+6 n_{5 b}+8 n_{7}=45$. It follows that $n_{2}=0$, and we have one of $\left(n_{1}, n_{5 a}, n_{5 b}, n_{7}\right)=(1,0,0,5),(0,1,2,3),(1,2,1,2),(2,3,0,1)$, $(0,3,3,0)$. These correspond to the graphs \#10, \#11, \#8, \#6 and \#9, respectively.

This settles the case of a path $P$ of length 6 where one of the components of $T \backslash P$ is of shape $\tilde{D}_{5}$, or $\tilde{E}_{6}$, or $\tilde{D}_{4}$ (attached at a leaf). Remain the 
cases where all components of $T \backslash P$ are of shape $A_{1}, A_{2}$, or $\tilde{D}_{4}$ (attached at the center), and that where $T$ has diameter smaller than 6 .

The case of diameter 5 is handled like that of diameter 6: if $P$ has length 5 , then by Godsil's Lemma $T \backslash P$ has eigenvalues $\theta,( \pm 2)^{a-1}$, $( \pm 1)^{b-1}, 0^{c-1}$ for a single eigenvalue $\theta$. But then $\theta=0$ and the spectrum is $( \pm 2)^{a-1},( \pm 1)^{b-1}, 0^{c}$, so is known and integral again.

\section{References}

[1] A. E. Brouwer, http://www.win.tue.nl/ aeb/graphs/integral_trees.html

[2] A. E. Brouwer, A. M. Cohen and A. Neumaier, Distance-Regular Graphs, Springer-Verlag, 1989.

[3] Z. F. Cao, Integral trees of diameter $R(3 \leq R \leq 6)$ (in Chinese), Heilongjiang Daxue Ziran Kexue Xuebao 2 (1988) 1-3, 95.

[4] C.D. Godsil, Spectra of trees, Annals of Discrete Mathematics 20 (1984) 151-159.

[5] R. L. Graham, On a Diophantine Equation Arising in Graph Theory, Europ. J. Comb. 1 (1980) 107112.

[6] F. Harary and A. J. Schwenk, Which graphs have integral spectra?, In: Graphs and Combinatorics, Lecture Notes in Mathematics 406, Springer-Verlag, Berlin (1974), 45-51.

[7] P. Híc and R. Nedela, Balanced integral trees, Math. Slovaca 48 (1998) 429-445.

[8] P. Híc and M. Pokorný, On integral balanced rooted trees of diameter 10, Acta Univ. M. Belii Math 10 (2003) 9-15.

[9] P. Híc and M. Pokorný, There are integral trees of diameter 7, Univ. Beograd. Publ. Elektrotehn. Fak. Ser. Mat. 18 (2007) 59-63.

[10] R. Y. Liu, Integral trees of diameter 5 (in Chinese), J. Systems Sci. Math. Sci. 8 (1988), no.4, 357-360.

[11] A. J. Schwenk, Trees with integral eigenvalues, Problem 23 in: A collection of open problems, M. Capobianco, S. Maurer, D. McCarthy and J. Molluzzo (eds.), pp. 565-590 in: Proc. Second International Conference on Combinatorial Mathematics (A. Gewirtz and L. V. Quintas, Eds.), Ann. New York Acad. Sci. 319 (1980) $582-583$.

[12] Ligong Wang, Integral trees and integral graphs, Ph. D. thesis, Univ. Twente, 2005.

[13] M. Watanabe, Note on integral trees, Math. Rep. Toyama Univ. 2 (1979) 95-100.

[14] M. Watanabe and A. J. Schwenk, Integral starlike trees, J. Austral. Math. Soc. (A) 28 (1979) 120-128. 